\title{
Meta
}

Journal des traducteurs

Translators' Journal

\section{Éléments pour une histoire de l'interprétation simultanée en Israël}

\section{Francine Kaufmann}

Volume 43, numéro 1, mars 1998

La traduction et l'interprétation en Israël

Translation and Interpreting in Israel

URI : https://id.erudit.org/iderudit/003292ar

DOI : https://doi.org/10.7202/003292ar

Aller au sommaire du numéro

Éditeur(s)

Les Presses de l'Université de Montréal

ISSN

0026-0452 (imprimé)

1492-1421 (numérique)

Découvrir la revue

Citer cet article

Kaufmann, F. (1998). Éléments pour une histoire de l'interprétation simultanée en Israël. Meta, 43(1), 98-109. https://doi.org/10.7202/003292ar
Résumé de l'article

Cet article retrace l'histoire des débuts de l'interprétation simultanée en Israël. Évoquant les précurseurs de l'Antiquité (époques biblique et talmudique), il décrit les premières utilisations de l'interprétation dans l'État d'Israël des années 50, avant même le procès Eichman (1961), la consolidation de la profession, dans les années 60 , la formation des interprètes de l'époque pionnière et la formation universitaire donnée, depuis 1972, à l'Université Bar-Ilan (Ramat-Gan). 


\title{
ÉLÉMENTS POUR UNE HISTOIRE DE L'INTERPRÉTATION SIMULTANÉE EN ISRAËL
}

FRANCINE KAUFMANN

Université Bar-Ilan, Ramat-Gan, Israël

\begin{abstract}
Résumé
Cet article retrace l'histoire des débuts de l'interprétation simultanée en Israël. Évoquant les précurseurs de l'Antiquité (époques biblique et talmudique), il décrit les premières utilisations de l'interprétation dans l'État d'Israël des années 50, avant même le procès Eichman (1961), la consolidation de la profession, dans les années 60, la formation des interprètes de l'époque pionnière et la formation universitaire donnée, depuis 1972, à l'Université Bar-Ilan (Ramat-Gan).
\end{abstract}

\begin{abstract}
This article is a historical review of simultaneous interpretation in Israel. Starting with the precursors of Antiquity, it describes the early uses of interpretation in the State of Israel in the 1950s--even prior to the Eichman Trial (1961), the development of the profession in the 1960s, the early beginnings of interpreter training, and current university training provided since 1972 at Bar-Ilan University (Ramat-Gan).
\end{abstract}

L'histoire de l'interprétation simultanée en Israël reste à faire. Jusqu'à présent, seuls quelques articles, partiels et parfois personnels, ont été publiés ${ }^{1}$. Cette étude, réunissant des témoignages de collègues et s'appuyant sur les sources existantes, tentera de jeter les bases de ce qui mériterait d'être développé plus tard, dans une recherche systématique.

\section{LES PRÉCURSEURS DANS L'ANTIQUITÉ JUIVE}

\subsection{Les précurseurs bibliques}

Commençons par dire que l'interprétation simultanée en Israël est l'héritière d'une longue tradition d'interprétation consécutive ou chuchotée, dont nous avons quelques traces écrites dans les sources juives. La Bible, en effet, rapporte que lors de leur descente en Égypte pour acheter du grain, (il y a quelque 3500 ans), les fils de Jacob furent reçus par le vice-roi (qui n'était autre que leur frère Joseph) et que l'audience se déroula par l'intermédiaire d'un interprète : le mèlits : "Et ils ne savaient pas que Joseph comprenait, car le mèlits était entre eux" (Genèse XLII,23). La racine du mot mèlits est phénicienne et désigne l'interprète. Mais ailleurs dans la Bible, le mot désigne aussi celui qui parle en bien, qui dit le bien, tel le mèlits yocher, sorte d'avocat de la défense (cf. Job XVI,20 et Job XXXIII,23). On comprend sans mal l'évolution sémantique du concept lorqu'on considère la fonction qu'occupait Aaron auprès de son frère Moïse. Selon la tradition, Moïse était bègue, et c'est le prétexte qu'il invoque pour se récuser lorsque Dieu le charge d'aller parler aux Hébreux et au Pharaon :

"De grâce Seigneur, je ne suis pas habile à parler [...] car j'ai la bouche pesante et la langue embarrassée" (Exode IV,10).

Meta, XLIII, 1, 1998 
Dieu lui adjoint alors un 'interprète' :

"Eh bien! ton frère Aaron, le Lévite, je sais que lui il parlera! [...] Tu lui parleras, et tu transmettras les paroles à sa bouche [...] il parlera pour toi au peuple; il te servira de bouche, et tu lui serviras de el (dieu)" (Exode IV, 14 à 16).

Il est probable qu'Aaron s'adressait en hébreu au peuple d'Israël et en égyptien au Pharaon. Mais sa fonction essentielle consistait moins à traduire qu'à réexprimer de manière éloquente ce que son frère Moïse, handicapé du langage, n'aurait pu formuler de manière convaincante.

\subsection{Les précurseurs de l'époque talmudique}

Cette distinction entre le locuteur qui sait et qui guide, et entre celui dont le talent d'orateur permet de transmettre avec conviction et clarté la doctrine dont il n'est que le porte-parole ("il te servira de bouche") se retrouve dans la fonction du metourguemane, 'l'interprète' évoqué dans le Talmud (vaste compilation rabbinique qui rapporte des faits s'étendant sur une dizaine de siècles, bien que le Talmud lui-même ait été rédigé entre le $\mathrm{II}^{\mathrm{e}}$ et le $\mathrm{VI}^{\mathrm{e}}$ siècle après Jésus-Christ). Certes, le metourguemane (ou tourguemane, d'une racine akkadienne qu'on retrouve aussi en ougaritique puis en araméen, et qui signifie: traducteur) est d'abord celui qui effectue le transfert d'un message d'une langue dans une autre.

Grâce au Talmud de Babylone, on sait, par exemple, que les tribunaux de Judée employaient des interprètes qui assistaient les accusés, sauf dans les cas particulièrement graves, notamment lorsque la peine de mort pouvait être requise, auquel cas on devait nommer au moins deux juges parlant les langues étrangères et un autre les comprenant (TB Sanhedrin 17 b). On trouvait aussi des interprètes au Temple de Jérusalem. Le Talmud palestinien vante un certain Peta'hia, (préposé aux oiseaux destinés aux offrandes), ainsi nommé car il clarifiait ('ouvrait'= pata' $h$ ) les paroles obscures des pèlerins : il connaissait, dit-on, non seulement les langues étrangères mais aussi le langage des sourds-muets. Et surtout il pouvait réexprimer, 'en clair', les propos des femmes ignorantes, qui ne savaient pas s'expliquer de manière cohérente et précise, ou qui parlaient en dialecte (TJ Cheqalim $\mathrm{V}, 1)$.

Mais c'est surtout dans l'usage liturgique et pédagogique que la fonction du metourguemane s'est développée. Car il était de règle, dès l'époque du Second Temple (soit depuis le cinquième ou le quatrième siècle avant l'ère vulgaire) de lire le texte hébreu de la Bible, accompagné de sa traduction orale en langue vernaculaire. Cette tradition se perpétua jusqu'au XII ${ }^{\mathrm{e}}$ siècle dans la plupart des communautés juives et elle s'est conservée jusqu'à ce jour chez les Juifs yéménites (Shinan 1993 : 13, 19, 29-30). Dans cette situation, le metourgueman se tient debout, aux côtés du lecteur, et interprète pour le public, en langue vernaculaire, le texte biblique verset après verset (selon des modalités longuement détaillées ailleurs : cf. Kaufmann 1995a et à paraître).

Enfin, on trouvait des interprètes dans les écoles et les académies talmudiques (fonction pédagogique). Ils étaient souvent attachés à la personne d'un rabbin dont ils avaient été le disciple et dont ils connaissaient la pensée et les enseignements. Certains semblent avoir été aussi des travailleurs "indépendants", auxquels on pouvait faire appel ponctuellement, dans des circonstances importantes (ainsi, pour la cérémonie d'investiture d'un nouveau juge au Sanhédrin). Dans les synagogues, ils étaient employés également pour réexprimer, à voix haute, la deracha du rabbin (sermon ou enseignement). La plupart sont restés anonymes mais on connaît quelques interprètes célèbres dont 'Houtspith ha-metourguemane, l'interprète de Rabban Gamliel, le directeur de l'Académie talmudique de Yabné, qui mourut en martyr sur le bûcher (à l'époque des persécutions d'Hadrien, au II 
siècle). Certains devinrent eux-même rabbins, ou du moins décisionnaires (on leur attribue en effet des lois ou des exégèses). Mais il semble que l'essentiel de leur fonction résidait moins dans la "traduction" (d'hébreu en araméen ou grec et vice-versa) que dans la réexpression éloquente, audible et bien composée, des paroles que le rabbin leur chuchotait à l'oreille (fonction du mélits).

Avec la dispersion du peuple juif en Occident et en Orient, les juifs servirent souvent d'intermédiaires linguistiques, notamment auprès des princes et des rois, grâce à leur connaissance des langues étrangères et des mentalités des pays dans lesquels leur commerce ou les tribulations de leurs familles (à la suite d'expulsions et de pogroms) les avaient fait séjourner ou s'établir. Là encore, nous manquons d'étude systématique pour attester la continuité de la fonction d'interprète et les modalités précises de leur travail. En tout cas, nous retrouvons de nombreux membres du peuple juif parmi les pionniers de l'interprétation moderne, dans les premières équipes de la S.D.N. et de l'O.N.U. et parmi les fondateurs de l'A.I.I.C.

\section{LES DÉBUTS DE L'INTERPRÉTATION SIMULTANÉE EN ISRAËL}

Lorsque l'État d'Israël fut créé, le 14 mai 1948, l'interprétation simultanée, rendue possible par les développements de la technologie, venait à peine de naître. Certains considèrent à tort qu'elle est apparue aux procès de Nüremberg (novembre 1945-octobre 1946). De fait, des essais avaient déjà été tentés sur du matériel mis au point par la société I.B.M. dès 1927, à Genève (Bureau International du Travail de la S.D.N.), puis dans un premier congrès international (de physiologie, en 1935 à Léningrad; cf. Delisle et Woodsworth 1995 : 248).

\subsection{L'interprétation hébreu-arabe au parlement}

Les Israéliens croient de même que c'est le procès d'Adolf Eichmann, (ouvert à Jérusalem le 11 avril 1961) qui introduisit l'usage de la simultanée en Israël. En réalité ce ne fut que la première occasion, pour le grand public de la découvrir. Elle était déjà utilisée régulièrement, dès le début des années cinquante, à la Knesset, le parlement israélien qui comptait, depuis sa création en 1949, quelques députés d'expression arabe. Certains d'entre eux ne comprenaient pas suffisamment l'hébreu pour pouvoir suivre les débats. C'est pourquoi un système d'interprétation simultanée de l'hébreu vers l'arabe fut mis en place. Deux interprètes permanents furent employés jusqu'en 1970, une cabine vitrée ayant été bientôt installée, donnant sur la plénière du parlement. Puis, après la guerre des Six jours, les députés arabes, maîtrisant de mieux en mieux l'hébreu et préférant parler hébreu en séance pour être compris 'en direct', un seul interprète suffit jusqu'en 1980, date à laquelle l'interprétation parlementaire en arabe fut supprimée et remplacée par des prestations ponctuelles ${ }^{2}$.

\subsection{L'interprétation dans les institutions sionistes}

Le second domaine d'utilisation des interprètes fut le Congrès Sioniste qui, depuis 1951, se tient tous les quatre ans à Jérusalem. Organe suprême de l'Organisation Sioniste Mondiale (l'O.S.M., créée en 1897 à Bâle) le Congrès Sioniste eut d'abord lieu dans les grandes villes d'Occident. Avant la guerre, la langue officielle y était l'allemand, ou plus précisément le Kongress Deutsch, (appelé aussi Konferenzdeutsch ou Conference German) une espèce de yiddish artificiellement rapproché de l'allemand, qui était compris sans trop d'effort par les germanophones, et qui permettait de se passer d'interpétation. Mais dans les années quarante, un changement de statut imposa l'hébreu comme langue officielle (les procès-verbaux étant publiés en hébreu et non plus en allemand depuis le vingtième Congrès Sioniste, en 1937). Quatre langues 
principales de travail furent alors admises : l'hébreu, l'anglais, le français et l'espagnol (Ikan 1997 : 17). Très vite, le recours à l'interprétation simultanée permit de résoudre les problèmes de communication lors des séances plénières du Congrès mais aussi dans l'intervalle, lors des réunions annuelles du Comité d'Action Sioniste (Zionist General Council), ainsi que durant les travaux annuels de l'Assemblée générale de l'Agence Juive pour Israël (association sœur de l'OSM, fondée en 1929 à Zürich, lors du seizième Congrès sioniste). De fait, l'O.S.M. et l'A.J. sont les seules organisations internationales ayant leur siège en Israël, à Jérusalem, et justifiant la présence régulière d'interprètes ayant l'hébreu dans leur combinaison linguistique 3 .

\subsection{L'interprétation de conférence dans le cadre de la coopération internationale}

Israël sert aussi de pays d'accueil à de grandes conférences internationales et à des congrès professionnels. Les premières manifestations semblent avoir eu lieu dans la seconde moitié des années 50. Ruth Lévy (éduquée à 'Haïfa dans la Palestine mandataire et diplômée de l'École d'Interprètes de Genève en 1952) se souvient d'une petite annonce parue dans la presse en 1956 pour recruter des interprètes. Elle émanait d'Adam Richter, lui aussi diplômé de l'École de Genève (à la fin des années 40), véritable pionnier de l'interprétation en Israël puisqu'il forma les premières équipes du Congrès Sioniste, dès $1951^{4}$.

Les premières conférences portèrent essentiellement sur la coopération internationale en matière agricole. En 1956 se tiennent successivement, en Israël, une grande conférence internationale du Mediterranean Citrus Board, (cultivateurs d'agrumes, langues de travail : EN-FRESP) et une première réunion d'une commission de la FAO sur l'élevage des moutons) $)^{5}$, bientôt suivie par d'autres, sur les problèmes de pâturage et de fourrage et d'une première réunion du Conseil de l'Europe ${ }^{6}$. Puis viennent les célébrations du dixième anniversaire de l'État d'Israël, en 1958 et les manifestations internationales commencent à devenir plus nombreuses. À cette époque, on fait venir de Londres Teddy Pilley, (l'un des fondateurs de la profession et de l'A.I.I.C.), établi comme interprète à Londres depuis 1936. Avec Adam Richter, Teddy Pilley recruta des candidats qu'il forma sur le tas. Raphaël Cidor, journaliste de radio à la section française de Kol Israël, se souvient de ce jour de 1958 où Adam Richter lui téléphona dans son bureau :

"M. Cidor ? M. Pilley et moi vous avons entendu sur les ondes : bonne voix, bon français. Vous pourriez faire un bon interprète. Nous avons monté des cabines pour nos candidats. Venez voir"7.

Raphaël Cidor refusa cette année là, mais il débuta avec Adam Richter en 1960, lors d'une importante conférence scientifique internationale qui se tint à l'Institut Weizman de Re'hovoth. Il y en eut plusieurs par la suite, qui réunissaient tous les deux ans des personnalités de premier plan du monde scientifique et politique. Ces conférences s'inscrivaient dans le cadre de l'aide agricole (internationale et israélienne) aux pays en voie de développement (Afrique et Asie) et durèrent jusqu'en 1967, c'est-à-dire jusqu'à la rupture des liens diplomatiques avec Israël par la plupart des pays du Tiers-Monde, à la suite de la guerre des Six Jours.

Parallèlement, des stages furent organisés par le ministère israélien de l'Agriculture pour des techniciens de pays en voie de développement. Les stagiaires venaient d'Afrique, d'Asie ou d'Amérique latine. Ils comprenaient le français, l'anglais ou l'espagnol, tandis que les enseignants et les moniteurs ne parlaient que l'hébreu (parfois l'anglais). Pendant des années, se souvient Shlomo Gitaï, plusieurs institutions israéliennes, dont le ministère des Affaires étrangères et le ministère de l'Agriculture, firent appel régulièrement à des interprètes pour pouvoir mener à bien leurs stages, donnant ainsi du travail plusieurs mois par an à une douzaine de personnes. Cela fournit à plusieurs débutants l'occasion d'acquérir une ample expérience ${ }^{8}$. 


\section{LE PROCÈS EICHMAN}

En 1960, après une conférence internationale qui s'était tenue l'été sur la formation et l'orientation professionnelle, les pionniers de l'interprétation en Israël travaillent, fin 1960 pour une conférence de l'International Union of Local Authorities (IULA). L'allemand y est l'une des langues de travail. On pouvait remarquer dans la salle, se souvient Ruth Lévy, un nombre inhabituel d'officiers de police, écoutant attentivement les interprètes. Ils étaient venus étudier le fonctionnement de l'interprétation et sélectionner des candidats pour le procès du criminel de guerre nazi, Adolf Eichman, qui devait commencer quelques mois plus tard. C'est le procès Eichman qui, en Israël, révèle l'interprétation simultanée et consécutive au grand public, grâce aux retransmissions en direct à la radio et aux reportages des actualités cinématographiques (la télévision israélienne n'ayant été fondée qu'en mai 1968). Le procès s'ouvre le 11 avril 1961, dans les locaux de la "Maison du Peuple", le Beyt Haam à Jérusalem (rebaptisé plaisamment par les interprètes : le "Eichmangrad", la forteresse Eichman tant les mesures de sécurité y sont draconiennes). Trois équipes différentes travaillent dans l'enceinte du bâtiment : l'une en consécutive, dans la salle d'audience, traduisant en hébreu pour la Cour, les interventions de l'accusé, Adolf Eichmann, et de son avocat, Maître Servatius, ainsi que les déclarations des témoins s'exprimant en langues autres que l'hébreu (russe, hongrois, yiddish, par exemple). Les trois juges maîtrisent parfaitement l'allemand et le Président du Tribunal, Moché Landau, contrôle (et reprend) le ou les interprètes. La seconde équipe travaille en simultanée et interprète, en cabine, tout ce qui se dit dans le prétoire, pour les besoins de l'accusé et de son défenseur. Les interprètes de ces deux équipes sont dûment assermentés et placés directement sous l'autorité de la Cour. La troisième équipe traduit l'ensemble des séances vers l'anglais et le français pour les très nombreux correspondants étrangers venus assister au procès. Quant aux journalistes d'expression allemande, nombreux eux aussi, ils peuvent suivre l'interprétation allemande de la deuxième équipe ${ }^{9}$. Le retentissement international, considérable, incite à enregistrer l'interprétation sur bande magnétique, dans toute les langues. Les bandes sont décryptées mais les tapuscrits, à la demande des interprètes, portent sur la première page la mention : "unedited transcript of simultaneous interpretation"10. Avant que le verdict ne soit prononcé, une partie des interprètes est chargée de traduire par écrit les attendus du tribunal et le texte de la sentence ${ }^{11}$. Enfermés durant dix jours dans le "Eichmangrad", ne rentrant chez eux que pour dormir, les traducteurs sont tenus au secret absolu. Répétons-le, ce procès, qui dura des mois (avril à décembre 1961, se soldant par la pendaison d'Eichman en avril 1962), et bouleversa les Israéliens en réveillant les douloureux souvenirs de la Shoa leur permit de découvrir les deux versants de l'interprétation orale : consécutive et simultanée.

Une génération plus tard, un second procès jouera un rôle similaire, celui de Ivan (John) Demjanjuk (16 février 1987 au 25 avril 1988) ${ }^{12}$. Nous n'en parlerons pas ici puisque nous pouvons renvoyer le lecteur aux études déjà publiées de nos collègues Ruth Lévy-Berlowitz (1989), la seule interprète à avoir travaillé dans les deux procès, Ruth Morris (1989b, c et d, 1990a) et Miriam Shlesinger (1989c, 1991).

\section{LES ANNÉES 60}

Après le procès Eichman, les occasions de travail commencent à se multiplier et à se diversifier. En 1963, une équipe israélienne se rend même pour la première fois à l'étranger pour participer, en Grèce, à un congrès de proctologie (EN, FR, G); d'autres contacts auront lieu avec la Grèce dans les années qui suivent. En juillet 1963, l'Office européen du vin et de la vigne tient son congrès en Israël (FR-EN-G). Les réunions de la FAO se pour- 
suivent; c'est aussi l'époque des contacts bilatéraux et multilatéraux : par exemple, entre le parti Mapaï et ses homologues socialistes européens.

Quant aux Israéliens, ils trouvent une nouvelle occasion de se familiariser avec la profession d'interprète lors des "Concours bibliques" qui passionnent alors le public. (Il y en aura trois dans les années 60 , retransmis en direct à la radio.) Les concurrents viennent des quatre coins du monde et il faut assurer l'interprétation des questions et réponses vers des langues européennes, scandinaves, ainsi que quelques langues plus rares, tel l'amharique, pour les candidats d'Éthiopie. Les chefs d'équipe déployèrent des trésors d'imagination pour découvrir des candidats capables, avec une formation minimale, d'assurer une interprétation digne de ce nom. Cette pléiade d'interprètes fit sur le grand public une impression profonde. Mais le nombre important de langues utilisées exigea de renoncer à la simultanée pour choisir la consécutive, ce qui ne permettait pas aux candidats de suivre le déroulement du concours lorsqu'il ne se tenait pas dans leur langue. Par ailleurs, les concurrents étaient à la merci du talent de leurs interprètes respectifs, deux pour chaque langue, l'un assurant la traduction de l'hébreu vers la langue du candidat, l'autre en sens inverse et les demandes de clarification ralentissaient le concours qui devint vite un dialogue entre les interprètes et le jury. La méthode fut ridiculisée dans un sketch particulièrement hilarant (interprété par deux super-vedettes de l'époque, Ouri Zohar et Arik Einstein). Diffusé à la radio et inclus dans le film d'Ouri Zohar Loul 4, ce sketch incita peut-être les organisateurs à repenser la logistique linguistique des concours bibliques. Quoi qu'il en soit, le concours changea de forme pour s'adresser uniquement à des adolescents juifs d'Israël et de la Diaspora. Il devint annuel et la finale, retransmise en direct à la radio et à la télévision, se déroule traditionnellement durant la matinée du jour de l'Indépendance. Mais les candidats reçoivent les questions traduites par écrit et doivent répondre dans l'une des langues connues des membres du jury. Il y eut pourtant quelques tentatives d'utiliser à nouveau l'interprétation simultanée dans des concours internationaux sur la résistance juive durant la seconde guerre mondiale et sur les mouvements clandestins juifs en Palestine ${ }^{13}$.

\section{LA FORMATION DES INTERPRÈTES}

\subsection{La constitution de la 'Région Israël' (mars 1968)}

La nécessité de former des interprètes professionnels s'était rapidement fait sentir, dès les années 50. La génération des pionniers était, en partie, diplômée de l'École de Genève et des nouveaux immigrants, interprètes formés notamment à Bruxelles, Londres et Genève, vinrent renforcer les rangs des 'professionnels', dans les années 70 et 80 . Dès 1968, le nombre d'interprètes locaux atteignit les douze membres actifs statutairement nécessaires pour demander une adhésion en bloc à l'AIIC. Privés de contacts avec leurs collègues des pays voisins (par suite du conflit israélo-arabe), ils demandèrent à être admis comme 'région', ce que l'assemblée de l'AIIC ratifia lors de sa quinzième session ordinaire (16-17 mars 1968).

Vingt ans plus tard, on compte dans les rangs de l'AIIC - Israël, 23 membres inscrits dans l'annuaire de 1998 (sans compter les candidats), avec des combinaisons qui, outre l'hébreu et l'anglais, indispensables, présentent essentiellement le français (17), l'espagnol (10), l'allemand (7), le yiddish (3), l'italien (3), et depuis peu le russe (1).

\subsection{L'époque héroïque}

La multiplication des congrès spécialisés, les progrès technologiques, la banalisation du recours à l'interprétation et le développement des échanges internationaux où, malgré le boycottage arabe, Israël prenait une place de plus en plus importante, avait donc exigé une professionnalisation accrue de l'interprétation en Israël. Si, dans les années 50, les pionniers 
s'étaient contentés d'asseoir les candidats dans des salles de congrès, avec des écouteurs et des recommandations assez vagues, avant de les 'essayer' dans les congrès juifs puis de les lâcher dans l'arène, aux côtés d'un collègue expérimenté, les normes nouvelles (surtout depuis le dépôt de la candidature d'adhésion à l'AIIC) exigeaient de rationaliser la formation. Dès les années 60, après le procès Eichman, une nouvelle génération d'Israéliens commençait d'ailleurs à songer à faire de l'interprétation sa profession. L'infatigable Adam Richter entreprit, dès le début des années 60 , de fournir une formation pratique accélérée (blocs de cinq à six mois) aux candidats (souvent recrutés par voie de presse et sélectionnés au cours d'un entretien préliminaire). Les locaux changeaient régulièrement (de l'appartement privé aux salles de classe louées dans des institutions d'accueil). Les enseignants (dont Adam Richter et Ruth Lévy-Berlowitz) étaient des interprètes chevronnés, auxquels s'ajouta une jeune Israélienne, Batya Frost, qui venait de terminer l'École de Genève (1958-1961) et put enseigner la traduction écrite en hébreu, sa langue maternelle ! Les autres enseignants étaient d'anciens immigrants qui avaient été recrutés (et souvent formés sur le tas par Richter lui-même). Outre la terminologie, les élèves pratiquaient tous les modes de traduction (écrite, consécutive, simultanée). Parmi les produits de cette formation, on compte certains des vétérans de la profession : Esther Guilon, Monique Neumark, Naomie Ofri, Shlomo Guitaï, Rivka Friedgurt, Ayeleth Nirpaz... Ayeleth, issue de la promotion 1971-1972 se souvient que les cours avaient eu lieu, cette année-là, dans les locaux du Beyt Tsioney América, à Tel-Aviv. La classe était composée de cinquante élèves, disposant de deux cabines où ils se relayaient (le reste de la classe étant équipés de casques et de transistors). Chacun travaillait vers sa langue maternelle, seule la langue de départ changeait : on interprétait donc à partir de l'hébreu, de l'anglais ou du français vers les autres langues (essentiellement d'anglais en hébreu, mais aussi en français, en allemand, en espagnol). Sur les cinquante élèves, seule Ayeleth Nirpaz est devenue interprète active (après être revenue s'entraîner, en 1972-1973, avec les élèves de la dernière promotion de cette catégorie de cours).

\subsection{L'École de traducteurs et interprètes de l'Université Bar-Ilan}

Au début des années 70, Adam Richter entreprit de faire le tour des trois principales universités du centre du pays (Jérusalem, Tel-Aviv et Ramat-Gan) pour tenter d'imposer l'idée d'une formation universitaire à la traduction et à l'interprétation, dans l'esprit des formations qui commençaient à se multiplier, notamment en Europe. Il avait établi un programme qu'il proposait d'adapter aux réalités israéliennes. Après bien des négociations, c'est finalement l'Université Bar-Ilan (située à la lisière des villes de Ramat-Gan et de Bné-Brak, dans la plaine côtière, non loin de Tel-Aviv) qui accepta le projet.

Dès l'année universitaire 1972-1973, une section hébreu-anglais fut créée, à laquelle s'ajouta, à la rentrée 1974, une section française. Malgré les tentatives de créer une section arabe, le projet n'aboutit pas, par manque de candidats de niveau suffisant. Plusieurs sessions d'examen d'entrée eurent lieu à des époques différentes, et il arriva que des cours de traduction écrite, hébreu-arabe, puissent être dispensés, notamment dans les années 80. De même, des sections espagnole et allemande fonctionnèrent entre 1989 et 1996, mais elles sont gelées actuellement jusqu'à ce qu'un nombre suffisant de candidats de valeur soient réunis. Le même problème empêche l'ouverture d'une section russe, projetée depuis quelques années.

'L'École' (baptisée modestement: Hamegama limetourguemanoute, c'est-à-dire 'l'option de traduction', pour ne pas dire département ou école) est, aujourd'hui encore, la seule du pays à former des interprètes professionnels (et bien sûr la seule au monde à former des interprètes possédant un hébreu actif et rompus à la terminologie spécifique du marché israélien). Les études durent deux ans et portent sur un seul couple de langues (hébreu et langue étrangère). Mais les étudiants ont la possibilité d'obtenir, en trois ans, un double diplôme, portant sur trois langues. Il peuvent également compléter leur diplôme et obtenir une maîtrise 
en un an auprès des départements d'anglais et de français de Bar-Ilan. Un projet de maîtrise en traduction a été soumis très tôt aux autorités universitaires, mais des réticences diverses ont retardé l'acceptation du projet, bien que depuis 1995, Bar-Ilan ait donné son accord de principe.

\subsection{Les modalités de la formation à Bar-Ilan}

Le programme d'études de la megama s'adresse à des étudiants qui justifient d'un diplôme de premier cycle (licence, B.A. ou équivalent) et passent avec succès l'examen d'entrée (écrit et oral) destiné à contrôler leurs connaissances linguistiques (actives et passives), leur culture générale et leur motivation. Les candidats doivent posséder, outre l'hébreu (langue pivot), au moins l'une des langues enseignées (et l'anglais de surcroît).

L'enseignement théorique (relativement réduit) initie les étudiants à la réflexion sur les problèmes liés à la traduction (linguistique, sociolinguistique, théories de la traduction, stylistique appliquée à la traduction, grammaire contrastive...). L'enseignement pratique, outre des cours à option qui changent régulièrement, comporte la traduction écrite ainsi que la préparation à l'édition, et l'interprétation simultanée et consécutive, qui sont obligatoires dès la première année pour tous les étudiants, même ceux qui ne souhaitent pas s'y consacrer dans leur activité professionnelle. Un diplôme de traducteur est décerné aux étudiants ayant atteint au cours de leurs études la moyenne de notes requise, mais seuls obtiennent un diplôme d'interprète les candidats ayant subi avec succès les épreuves d'interprétation par devant un jury d'enseignants et d'interprètes actifs auquel s'ajoutent des examinateurs extérieurs.

Pour exister, Bar-Ilan a dû tenir compte des contraintes spécifiques d'un pays à la superficie certes réduite (à peine plus de $20000 \mathrm{kms}$ carrés, dont la moitié de désert) mais comme la zone peuplée s'étend sur une bande étroite qui longe le littoral du nord au sud, les étudiants viennent parfois de très loin pour étudier. La population d'Israël (un peu plus de trois millions début 1970, près de six millions aujourd'hui, en comptant le million d'immigrants russes et la population arabe) est souvent plurilingue mais à peine deux millions et demi d'Israéliens possèdent, en 1998, l'hébreu comme langue maternelle. (Ils étaient moitié moins nombreux à la création de l'école.) De surcroît, l'enseignement des langues étrangères autres que l'anglais et l'arabe au niveau du lycée est peu développé et les départements universitaires de langues ont eux-mêmes des problèmes de recrutement. (Au niveau de la licence, il existe en Israël trois départements de français, un d'allemand et un d'espagnol). Enfin, la difficulté, dans un pays d'immigration, de trouver des candidats possédant une 'vraie' langue maternelle et ayant fait des études suivies et approfondies dans cette langue complique le travail de sélection et lorsque plus de la moitié, voire les deux-tiers, des candidats ont été éliminés à l'examen d'entrée, les candidats retenus dans une section sont à peine assez nombreux pour peupler une classe digne de ce nom. C'est pourquoi, afin de ne pas subdiviser encore les effectifs réduits, TOUS les étudiants d'une section suivent ensemble TOUS les cours obligatoires de la section : les hébréophones sont ainsi mêlés aux locuteurs de langue étrangère, les élèves traducteurs aux élèves interprètes. Curieusement, cette situation née de contraintes pratiques, n'a pas que des inconvénients. Les hébréophones tirent parti de la présence des locuteurs de langue étrangère (et vice-versa) dans les cours de traduction écrite, et les cours de simultanée (et de consécutive) enrichissent le réservoir terminologique des futurs traducteurs et leur apprennent à moins coller aux mots et à voir le texte de manière globale (comme le fait le bon interprète).

Signalons encore que tous les membres du corps enseignant des cours d'interprétation sont des interprètes actifs, membres de l'AIIC, et certains sont eux-mêmes anciens élèves de l'école. Au cours des quelque vingt-cinq années de son existence, l'école a formé près de la moitié des interprètes travaillant aujourd'hui en Israël et elle est homologuée par le Comité de formation de l'AIIC. 
La recherche dans le domaine de l'interprétation simultanée en Israël en est à peine à ses débuts. Les quelques travaux de recherche faits jusqu'ici ont été réalisés (comme on pouvait s'y attendre) par des enseignants de l'École d'interprètes et traducteurs de l'Université Bar-Ilan (cf. bibliographie, ci-dessous). Par ailleurs, des rencontres périodiques ont lieu depuis 1996, dans le cadre de la région Israël de l'AIIC, pour débattre de certains aspects de la recherche théorique en interprétation.

\section{6. ÉTAT DES LIEUX}

Depuis les années 70, la pratique de l'interprétation en Israël s'est banalisée et le métier ressemble, par bien des aspects, à ce qu'il est ailleurs. Certes l'exiguïté du marché, l'enfermement géographique par suite du conflit israélo-arabe, explique que les débouchés soient limités : les interprètes israéliens, aussi bons soient-ils, ne peuvent s'insérer dans leur cadre régional naturel, le Moyen-Orient, et doivent (sauf s'ils ont des contacts professionnels outremer) se contenter des conférences qui s'organisent en Israël. Par ailleurs, en période de tension (plus nettement après la guerre des Six jours et surtout depuis le début de l'Intifada, la guerre des pierres, dans les territoires administrés par Israël), de nombreuses organisations internationales, professionnelles et non gouvernementales évitent de tenir leurs assemblées ou congrès en Israël par crainte d'un absentéisme massif dû au boycottage d'Israël par les pays arabes (partiellement suspendu au début du processus de paix) ainsi qu'à une peur (largement exagérée) d'actes de terrorisme ou de guerre. Si l'on ajoute à ces conditions géopolitiques les conditions économiques qui font que la plupart des Israéliens touchent encore des rémunérations proches de celles du Tiers-Monde tout en fournissant des services du niveau des pays hautement développés, on ne s'étonnera pas que les cachets versés aux interprètes pour leurs prestations soient parmi les plus bas de ceux pratiqués dans les pays où l'AIIC est présente. Les conditions de travail qui leur sont offertes ne sont pas non plus du niveau de celles de leurs collègues européens. Rien d'étonnant donc si les interprètes israéliens sont tenus d'avoir une profession régulière (ce qui exige des prouesses d'organisation en période de congrès) et s'il leur faut plusieurs années avant de pouvoir justifier des 200 journées de travail exigées par l'AIIC ${ }^{14}$. La jeune génération israélienne est consciente des inconvénients matériels de la profession malgré le prestige incontestable dont elle jouit en Israël (d'où le nombre limité de candidats).

Enfin, les hauts dignitaires de l'État rechignent à employer l'hébreu dans leurs rencontres avec des chefs d'État et des personnalités politiques étrangères, voire même dans les grandes manifestations internationales où ils préfèrent parler l'anglais, pour être compris 'en direct' (comme la conférence de Madrid en novembre 1991 ou la remise du prix Nobel de la paix à Yits'hak Rabin et Shimon Pérès, en mai 1996). Même si ce n'est pas spécifique à Israël, l'anglais s'y est donc imposé comme langue internationale véhiculaire, au point que le dernier ministre des Affaires étrangères, M. David Lévy, qui parlait un français plus qu'honorable était considéré comme ne parlant pas de langues étrangères (parce qu'il ne savait pas l'anglais) et que le Président Ezer Weizman a fait sensation en décidant, ostensiblement, de prendre la parole en hébreu au Bundestag, lors de sa visite officielle en Allemagne (janvier 1996, l'interprète était Batya Frost). L'exemple, donné de haut, est suivi dans de nombreuses manifestations officielles et dans de nombreux congrès, universitaires et professionnels, où l'essentiel des débats se déroule en anglais (donc, sans interprètes).

Malgré toutes ces difficultés, la profession d'interprète reste très recherchée, en Israël. Étant donné l'exiguïté du marché, la concurrence est sévère et seuls les meilleurs s'imposent. La qualité de l'interprétation en bénéficie. Le recours à des interprètes dans des festivals de cinéma, de théâtre ou à l'opéra, lors des tournées à l'étranger de théâtres comme Habima ou le Camri, parfois dans les médias (surtout pour les grands événements politiques) font que les 
interprètes israéliens restent présents dans la conscience du grand public, et pas seulement dans les salles confidentielles des congrès internationaux.

Les débuts prometteurs du processus de paix avaient pu laisser rêver un instant à un nouveau Moyen-Orient, où les interprètes pourraient voyager et devenir des ambassadeurs de la communication pacifique. Mais l'assassinat de Yits'hak Rabin (4 novembre 1995) a repoussé à plus tard les rêves d'une normalisation prochaine de la situation. Dans l'intervalle, les interprètes continuent à faire leur travail quotidien en permettant l'établissement de liens plus fraternels, levant les barrières linguistiques qui, sans eux, compliqueraient encore les relations d'Israël avec le monde ${ }^{15}$.

\section{Notes}

1. Voir notamment, dans les références, les articles de Gitaï 1993; Kaufmann 1987, 1993a, 1993b, 1995b; Lévy-Berlowitz 1989; Morris 1989b, 1989c, 1989d, 1990a; Shlesinger 1989c.

2. Témoignages recueillis auprès de Victor Na'hmias (l'un des permanents) et de Marcelle Saguiv (free-lance, qui a vérifié ces données auprès de l'archiviste de la Knesset). La télévision n'ayant été introduite qu'en 1968 en Israël, les séances de la Knesset n'étant “vues" qu'aux actualités cinématographiques, on peut comprendre que le public israélien n'ait pas été conscient de l'emploi courant, au Parlement, de la simultanée dans les années 50.

3. Il est intéressant de signaler à ce propos que le yiddish, qui était encore langue officielle aux premiers congrès sionistes de l'après-guerre, en a complètement disparu depuis une vingtaine d'années (même comme langue passive). L'allemand, langue honnie après la Shoa (cf. ci-dessous note 5), n'est plus une langue de travail du Congrès. Le russe est introduit occasionnellement depuis l'afflux des juifs d'Union Soviétique, à la fin des années 80. Il faut encore noter que l'Agence juive tient généralement ses débats en anglais (avec interprétation en hébreu et dans une ou plusieurs langues des délégués...).

4. Juif polonais établi en Israël vers 1947-1948 et maîtrisant notamment l'hébreu, l'allemand, l'anglais et le français, Adam Richter est décédé en 1977. Parmi ses collaborateurs de la première heure, citons Yossi Rosen (après sept ans de travail à Londres pour le service hébreu de la BBC) et Bilha Mikhaëli, avocate (et diplômée de mathématiques). Éduquée dans la Palestine mandataire, Bilha Mikhaëli, disait à qui voulait l'entendre qu'elle souhaitait mourir en cabine. Agée de 80 ans, l'esprit encore vif et clair (mais devant être soutenue pour rejoindre sa cabine, car elle avait des difficultés de locomotion), elle ressentit un malaise durant un congrès technique, dût se faire remplacer et mourut le lendemain, (17 octobre 1990). Ses collègues vinrent à son enterrement directement du congrès qui se poursuivait sans elle.

5. La tenue de cette conférence posa un problème de société du fait que des délégués allemands y participaient. Peu après la Shoa (et durant de longues années), les Israéliens boycottèrent tout ce qui venait d'Allemagne, y compris la langue allemande et la musique de Wagner. Pour la réunion de la FAO, qui se tint à Tel-Aviv, à la ZOA House (Beyt Tsioney América, Maison des Sionistes américains), on décida de ne hisser aucun drapeau pour éviter de devoir faire figurer un drapeau allemand parmi ceux des nations participantes. (Souvenirs de Ruth Lévy qui interprétait en chuchotage pour un grand propriétaire terrien allemand, le Baron von Wülfing.)

6. Rejeté politiquement par l'Asie et l'Afrique (à la suite du boycottage arabe), Israël appartient à la région Europe pour l'ensemble des travaux des institutions spécialisées de l'ONU. Il est observateur au Conseil de l'Europe.

7. Raphaël Cidor, conférence donnée à l'Université Bar-Ilan le 15 novembre 1979, p. 10 (documents de l'auteur). Sur le mode de recrutement par Adam Richter et par son associé, Yossi Rosen, voir Gitaï (1993 : 674) et Kaufmann (1993a : 659-660). Parmi les interprètes formés sur le tas, il faut noter à ce propos la place particulière (est-ce un phénomène spécifiquement israélien ?) des interprètes recrutés parmi les journalistes de radio (essentiellement Kol Israël, la radio israélienne qui possède des services en langues étrangères). Parmi eux citons notamment : cabine anglais-hébreu : Yossi Rozen (service hébraïque de la BBC à Londres), Gad Lévy; cabine française : Raphaël Cidor, Jean Slavic, 'Hanan Avriel, Francine Kaufmann, (et Michel Zlotovski, devenu interprète après être reparti en France); cabine espagnole : Shlomo Gitaï, Rivka Friedgurt, Avi Nadra; cabine russe : Victor Radutsky.

8. L'interprétation de liaison dans les stages se réduisit peu à peu, lorsque les clients, soucieux d'efficacité (et de rentabilité) commencèrent à recruter des instructeurs s'exprimant plus ou moins bien dans les langues des stagiaires, ce qui permit de supprimer les interprètes. Depuis le début des années quatre-vingt le travail dans ce domaine a presque complètement cessé (témoignage de Shlomo Gitaï). Notons qu'un diplômé de l'École d'interprètes de Bruxelles (Michel Isaac) a été engagé comme fonctionnaire au ministère de l'Agriculture.

9. Voici la composition des équipes selon le témoignage de Ruth Lévy : Première équipe : pour la Cour, allemand-hébreu en consécutive dans la salle du prétoire : Yossi Rosen (remplacé par la suite par un policier germanophone, formé sur le tas), en cabine allemande: Ruth Lévy et Benno Lichtenberg, (un avocat, et comme remplaçante, une critique d'art: Myriam Tal). Deuxième équipe : pour la presse : cabine anglaise : Bilha 
Mikhaeli et Adam Richter (à un moment : Gad Lévy). Cabine française : Raphaël Cidor et Roxanne Meron (diplômée de Genève ?, devenue par la suite permanente à l'ONU, aujourd'hui retraitée). Pour tenir nerveusement, malgré l'extrême horreur des témoignages réexprimés, les interprètes sortaient chaque soir pour boire et danser dans les rares bars de Jérusalem (Bacchus, Mandarine). La même stratégie avait été adoptée par les interprètes du Procès de Nüremberg, comme Ruth Lévy s'en rendra compte en écoutant leur témoignage lors de la séance du 28 août 1992, consacrée aux débuts de l'interprétation simultanée, durant l'assemblée extraordinaire de l'AIIC à Bruxelles. (Lire aussi l'interview de Adam Richter, dans le Jerusalem Post, 26/4/61).

10. Souvenirs de Ruth Lévy. Dans les années 80 : on établira une traduction officelle des protocoles.

11. Ruth Lévy et Benno Lichtenberg (allemand), Raphaël Cidor (français), Bilha Mikhaëli (anglais), ces deux derniers assistés de traducteurs (souvenirs de Ruth Lévy).

12. Sur l'imbroglio linguistique qui régnait durant le Procès Demyanjuk, voici une anecdote qui m'a été rapportée par Ilan Steinberg, l'un des interprètes (remplaçant en consécutive hébraïque l'interprète principale : Batya Frost) : les juges et la partie civile parlaient l'hébreu, l'accusé l'ukrainien, l'avocat de la défense l'anglais. On avait prévu une consécutive en hébreu, un chuchotage en ukrainien, et une simultanée en anglais. Le contre-interrogatoire se poursuivant indûment, le juge demanda à l'avocat quand il comptait terminer : - "Soon, your honor, very soon" - "Kama soon, zè soon ?" (Combien "soon" c'est "soon" ?) s'enquit le juge excédé, mêlant sans s'en rendre compte l'hébreu et l'anglais. (La phrase devint ensuite un refrain familier des interprètes en fin de journée.)

13. Ainsi la finale télévisée en direct du "First International Quiz on Jewish Underground Activities" (Tel-Aviv, 28/10/1981, en présence du Premier ministre, M. Menahem Begin) ou la finale qui s'était tenue à Varsovie, à l'issue de la première "Marche des Vivants", (avril 1989), finale retransmise en direct par satellite, l'équipe d'interprètes/ H/EN/FR/ESP) ayant été amenée d'Israël (en majeure partie).

14. La moyenne des journées de travail est d'environ trente à quarante par an pour les interprètes actifs, sauf s'ils pratiquent l'hébreu et l'anglais (avec le retour), seule combinaison véritablement rentable. Les débutants doivent se contenter, au mieux, d'une dizaine de journées de travail par an.

15. Je tiens à remercier tous les collègues et amis interprètes qui m'ont aidée à préparer cet article (Shlomo Gitai et Ruth Lévy), ou qui ont répondu à mes questions ou attiré mon attention sur des faits importants : Annette Dulczin, Batya Frost, Choulamith Haran, Arlette Kirshman, Ruth Morris, Ayeleth Nirpaz, Marcelle Saguiv.

\section{RÉFÉRENCES}

DELISLE, Jean et Judith WOODSWORTH (dir.) (1995) : Les traducteurs dans l'histoire, Ottawa, Les Presses de l'Université d'Ottawa et Paris, Éditions UNESCO. (Édition anglaise: Translators Through History, Amsterdam. John Benjamins et Paris UNESCO.)

GITAÏ, Shlomo (1993) : "Formé sur le tas", Meta, n spécial : Le "Je" du traducteur, sous la direction d'André Clas, 38 (4), pp. 674-676.

IKAN, Yael (1997): The World Zionist Organisation, The National Institutions. Structure and functions, Jerusalem, The Department of Organisation and Community Relations, WZO, $136 \mathrm{pp}$.

KAUFMANN, Francine (1987): "Traduire et interpréter", Bar-Ilan, ${ }^{\circ}$ 1, juin 4-6 (revue en français de l'Université Bar-Ilan; aussi dans Tribune juive, n ${ }^{\circ}$ 960, du 20 au 26 février 1987, pp. 14-16).

KAUFMANN, Francine (1988) : "La formation des traducteurs et interprètes en Israël”, Traduire, $\mathrm{n}^{\circ}$ spécial de la Revue des Universités francophones (AUPELF), Montréal, Canada, pp. 33-35.

KAUFMANN, Francine (1995a) : "Le traducteur et l'interprète juifs de la Bible", Les traducteurs dans l'histoire, Ottawa, Les Presses de l'Université d'Ottawa et Paris, Éditions UNESCO, pp. 165-169.

KAUFMANN, Francine (1993b) : "En équilibre entre deux mondes : un itinéraire de Paris à Jérusalem", Meta, n ${ }^{\circ}$ spécial : Le "Je" du traducteur, sous la direction d'André Clas, 38 (4), pp. 658-663.

KAUFMANN, Francine (1993a): "Interprétation consécutive et interview dans le film Shoah de Claude Lanzmann", Meta, 38 (4), pp. 664-673.

KAUFMANN, Francine (1994) : "Interpreters in Early Judaism", The Jerome Quarterly, Université Georgetown, USA, 9 (3), p. 2.

KAUFMANN, Francine (1995a) : "Formation à la traduction et à l'interprétation pour les médias audiovisuels", Yves Gambier (Ed.), Translatio, XIV (3-4), Actes du colloque de Strasbourg, Comité Médias de la FIT, EST, Conseil de l'Europe, pp. 431-443.

KAUFMANN, Francine (1995b) : "Place et perspectives de l'interprétation simultanée et consécutive dans les actualités radiophoniques", Yves Gambier (Ed.), Translatio, XIV (3-4), Actes du colloque de Strasbourg, Comité Médias de la FIT, EST, Conseil de l'Europe, pp. 363-375.

KAUFMANN, Francine (à paraître) : "La fonction de l'interprète synagogal dans l'antiquité juive", Target.

LÉVY-BERLOWITZ, Ruth (1989): "The linguistic logistics of the Demjanjuk trial", Parallèles, Cahiers de l'École de Traduction et d'Interprétation, Université de Genève, $\mathrm{n}^{\circ}$ 11, pp. 37-43.

LÉVY-BERLOWITZ, Ruth (1990) : "Interpreting in Multicultural Settings", Interpreting--Yesterday, Today and Tomorrow, Bowen \& Bowen (Eds.), American Translators Association Scholarly Monograph Series, Vol. IV, State University of New York at Binghamton (SUNY), pp. 117-121. 
MORRIS, Ruth (1988) : “Court Interpreting”, ITI News, Vol. 3, n 1.

MORRIS, Ruth (1989a) : "Court Interpreting” (Hebrew), Targima, Israel Translators Association Journal, $\mathrm{n}^{\circ} 10$, pp. 16-19.

MORRIS, Ruth (1989b) : "Shifts in Interpretation at the Demjanjuk Trial" (Hebrew), Hed ha-Ulpan, Israel Ministry of Education and Culture, Adult Education Division, $\mathrm{n}^{\circ}$ 57, pp. 103-108.

MORRIS, Ruth (1989c): "Court Interpretation: The Trial of Ivan John Demjanjuk--A Case Study", The Interpreters' Newsletter, Trieste, University School of Interpretation and Translation, $\mathrm{n}^{\circ}$ 2, pp. 27-37.

MORRIS, Ruth (1989d) : "Court Interpretation and the Record of Legal Proceedings : Eichmann v. Demjanjuk", Parallèles, Cahiers de l'École de Traduction et d'Interprétation, Université de Genève, n 11, pp. 9-28.

MORRIS, Ruth (1990a) : "Interpretation at the Demjanjuk Trial", Interpreting--Yesterday, Today and Tomorrow, Bowen \& Bowen (Eds.), American Translators Association Scholarly Monograph Series, Vol. IV, State University of New York at Binghamton (SUNY), pp. 101-107.

MORRIS, Ruth (1990b) : “Court interpreter : record or participant?”, Jovanovic, Mladen (Ed.), Proceedings, XIIth World Congress of FIT, Belgrade, Prevodilac, pp. 724-729.

MORRIS, Ruth (1992): “Conference Interpreting in a Single Market”, C. Picken (Ed.), Proceedings, ITI Conference, 6, London, Aslib, pp. 97-105.

MORRIS, Ruth (1993a): "Seminar on Interpreters and the Law -- Luxembourg, 11-13 January 1993", AIIC Bulletin, XXI/2, pp. 31-33.

MORRIS, Ruth (1993b) : “Justice for non-English speakers”, New Law Journal, Vol. 143, n 6610, pp. 10591060.

MORRIS, Ruth (1993c) : "Justice for non-English speakers-- Interpreters and the legal profession", Language International, 5/3, pp. 35-36.

MORRIS, Ruth (1993d) : "Justice for non-English speakers-- Interpreters and the legal profession", $L P L P$ (Language Planning and Language Policy), 17 (3), pp. 265-268.

MORRIS, Ruth (1993e) : "Nobs And Yobs--the Provision of Interpreters for Legal Proceedings involving Highstatus Foreigners and Others", C. Picken (Ed.), Proceedings, XIIIth World Congress of FIT, Institute of Translation and Interpreting, pp. 356-366.

MORRIS, Ruth (1993f) : “Justice for non-English Speakers -- Interpreters and the legal profession”, Translatio, New series XII, $n^{\circ} 1-2$, pp. 204-208.

MORRIS, Ruth (1993g) : “The Interlingual Interpreter--Cypher or Intelligent Participant? Or, The Interpreter's Turn..., International Journal for the Semiotics of Law, VI/18, pp. 271-291.

MORRIS, Ruth (1994) : Review of Cecilia Wadensj, "Interpreting As Interaction : On dialogue-interpreting in immigration hearings and medical encounters", Target, 6 (1), pp. 147-148.

MORRIS, Ruth (1995a) : "English as she is spoke..." (Hebrew), Targima, n 33, pp. 12-13.

MORRIS, Ruth (1995b) : "NAS workshop on court interpreting, Sharm El Sheikh, 4 January 1995”, AIIC Bulletin, XXIII/1, p. 41.

MORRIS, Ruth (1995c) : "The Moral Dilemmas of Court Interpreting”, The Translator, Vol. 1, n 1, pp. 25-46.

MORRIS, Ruth (1995d): "Pragmatism, Precept, and Passions: The Attitude of English-Language Legal Systems to Non-English Speakers", Translation and the Law, American Translators Association Scholarly Monograph Series, Morris, M. (Ed.), Vol. VIII, Amsterdam and Philadelphia, John Benjamins, pp. 263-279.

MORRIS, Ruth and Joan COLIN (1996) : Interpreting in the Legal Process, Winchester, Waterside Press.

MORRIS, Ruth (1997) : "Great Mischiefs -- A historical look at language legislation in Great Britain”, Language Legislation and Linguistic Rights, Amsterdam and Philadelphia, John Benjamins (forthcoming).

MORRIS, Ruth (1997) : "Technology and the Worlds of Interpreting”, Future and Communication : The Role of Scientific and Technical Communication and Translation in Technology Development and Transfer, San Francisco, London, International Scholars Publications, Rousenhouse, Bethesda, pp. 177-184.

SHINAN, Avigdor (1993): Mikra é'had vetargoumim harbé (The Biblical Story as reflected in its aramaic translations) (hébreu), Bibliothèque Hillel ben-'Hayyim, Tel-Aviv, Éditions Hakibbouts Hameou'had.

SHLESINGER, Miriam (1989a) : Simultaneous Interpretation as a Factor in Effecting Shifts in the Position of Texts on the Oral-Literate Continuum, Tel Aviv, Tel Aviv University. [M.A. thesis]

SHLESINGER, Miriam (1989b) : "Extending the Theory of Translation to Interpretation : Norms as a Case in Point”, Target, 1 (1), pp. 111-115.

SHLESINGER, Miriam (1989c): “Monitoring the Courtroom Interpreter", Parallèles, Cahiers de l'École de Traduction et d'Interprétation, Université de Genève, n 11, pp. 29-36. 